\title{
Simvastatin decreases the level of heparin-binding protein in patients with acute lung injury
}

\author{
Daniel F McAuley ${ }^{1,2}$, Cecilia M O'Kane ${ }^{2}$, Thelma R Craig ${ }^{1}$, Murali Shyamsundar ${ }^{1}$, Heiko Herwald ${ }^{3}$ and Karim Dib ${ }^{2 *}$
}

\begin{abstract}
Background: Heparin-binding protein is released by neutrophils during inflammation and disrupts the integrity of the alveolar and capillary endothelial barrier implicated in the development of acute lung injury and systemic organ failure. We sought to investigate whether oral administration of simvastatin to patients with acute lung injury reduces plasma heparin-binding protein levels and improves intensive care unit outcome.

Methods: Blood samples were collected from patients with acute lung injury with $48 \mathrm{~h}$ of onset of acute lung injury (day 0), day 3, and day 7. Patients were given placebo or $80 \mathrm{mg}$ simvastatin for up to 14 days. Plasma heparin-binding protein levels from patients with acute lung injury and healthy volunteers were measured by ELISA.

Results: Levels of plasma heparin-binding protein were significantly higher in patients with acute lung injury than healthy volunteers on day $0(p=0.011)$. Simvastatin $80 \mathrm{mg}$ administered enterally for 14 days reduced plasma level of heparin-binding protein in patients. Reduced heparin-binding protein was associated with improved intensive care unit survival.
\end{abstract}

Conclusions: A reduction in heparin-binding protein with simvastatin is a potential mechanism by which the statin may modify outcome from acute lung injury.

Trial registration: Current controlled trials: ISRCTN70127774

Keywords: Acute lung injury, Simvastatin, Heparin-binding protein, Inflammation, Neutrophils

\section{Background}

Acute lung injury (ALI), and its most severe form, the adult respiratory distress syndrome (ARDS) are inflammatory disorders which are commonly caused by the systemic release of cytokines and pro-inflammatory molecules [1]. In the United States, there are approximately 190,000 cases per year of ALI with an associated death of 74,500 per year [1]. ALI is characterized by alveolar epithelial and capillary endothelial barrier damage, resulting in exudation of protein-rich oedema fluid into the alveolar space, which causes functional impairment of the gas exchange [1]. Uncontrolled and excessive infiltration of inflammatory cells, in particular neutrophils and macrophages, is a hallmark of ALI. Neutrophils are the main contributor in the pathogenesis of ALI. Indeed, there is a correlation between the number of neutrophils

\footnotetext{
* Correspondence: k.dib@qub.ac.uk

${ }^{2}$ Centre for Infection and Immunity, Queen's University of Belfast, Belfast, United Kingdom

Full list of author information is available at the end of the article
}

in bronchoalveolar (BAL) fluid and the severity of lung injury [2] and persistence of neutrophils is associated with mortality [3]. Neutrophils contribute to lung tissue destruction in ALI by producing tissue damaging agents such as reactive oxygen species, and granule-derived proteases [1].

In ALI, extravasation of neutrophils into the alveolar space is associated with disruption of the endothelial barrier. The mechanisms by which neutrophils induce change in alveolar and capillary endothelial barrier permeability is not well known, but may involve, at least in part, heparin-binding protein (HBP).

HBP, also known as CAP37/azurocidin, is a $28 \mathrm{kDa}$ protein belonging to the serprocidin subgroup of the chymotrypsin-like proteases [4]. HBP is contained in azurophilic granules and secretory vesicles of neutrophils [5]. HBP is released upon fusion of azurophilic granules and secretory vesicles with the plasma membrane. The basal release of HBP is augmented upon $\beta 2$ integrindependent adhesion of neutrophils to the vascular 
endothelium during inflammation [4]. HBP is a multifunctional protein of inflammation that is responsible for a number of host response mechanisms to infection. HBP exhibits antimicrobial activity [6], induces recruitment of monocytes to the site of inflammation [7], and augments macrophage phagocytosis [8]. Besides its beneficial effects during the immune response, HBP is also a potent inducer of endothelial hyperpermeability [4]. For example, neutrophil degranulation and the associated release of HBP, can be triggered by the binding of fibrinogen-coated $\mathrm{M}$ protein of streptococcus pyogenes to $\beta 2$ integrins, which causes a toxic shock syndrome characterized by plasma leakage and multi-organ failure [9].

Statins are inhibitors of hydroxyl-methylglutaryl coenzyme A reductase, the enzyme which catalyses the second reaction in the mevalonate-dependent biosynthesis pathway. Statins are being investigated as a potential therapy for ALI. Statins attenuate lung injury in vivo in mice which have received aerosolized lipopolysaccharide (LPS) [10] and attenuate vascular leak and inflammation in murine inflammatory lung injury [11]. In humans, oral intake of simvastatin for 4 days attenuates the systemic inflammatory response to intravenous LPS [12]. We have recently shown that simvastatin reduces pulmonary inflammation in vivo in healthy volunteers who have inhaled a low dose of LPS [13]. In this human model of ALI, simvastatin reduced LPS-induced BAL neutrophilia, tumour necrosis factor $\alpha$, matrix metalloproteinases and C-reactive protein. In a recent randomized, double-blind trial of 60 patients with ALI (the HARP study), we showed that simvastatin induced modest improvement in non-pulmonary organ dysfunction [14]. The mechanisms by which simvastatin exerted its protective effect in the small single centre HARP study were unclear. We hypothesised that the levels of plasma HBP were elevated in patients with ALI and that the protective effects of simvastatin were mediated by reduced plasma HBP levels.

\section{Methods}

\section{Study population}

The ALI population used to carry out this study has been previously described in detail [14]. This was a single-centre, prospective, double-blind, randomized, placebo-controlled clinical trial in which patients with ALI were randomized to simvastatin $80 \mathrm{mg}$ or placebo (1:1) for up to 14 days.

Baseline patient demographics, severity of illness scores were collected at baseline [14]. Briefly, 60 mechanically ventilated patients with ALI admitted to the regional Intensive Care Unit (ICU) in the Royal Victoria Hospital, Belfast, Northern Ireland, were recruited to the study. Five healthy subjects were recruited by advertising to undergo plasma sampling. Screening consisted of history, physical examination, routine blood investigation, electrocardiogram and spirometry. Healthy subjects included 4 males and one female. Mean baseline of FEV1 (L) and FVC (L) were $4.2(0.5)$ and 5.2(0.75), respectively. The study was approved by the local research ethics committee, and written informed consent was obtained from the legal representative of the patient or the healthy individuals. The research was carried out in compliance with the Helsinki Declaration.

\section{Preparation of plasma samples}

Blood was collected in lithium heparin tubes (BectonDickinson, Plymouth, UK) and placed immediately on ice until processed. Plasma sampling for patients with ALI were performed at baseline, before study drug administration, and on day 3 and 7 as described [14]. Total cell count was determined using a haemocytometer.

\section{Measurement of HBP levels}

Concentrations of HBP in plasma were determined using a validated ELISA as described [5].

\section{Statistical analysis}

Data are expressed as median (interquartile range). Data were analyzed by unpaired t-test or Mann-Whitney U-test as appropriate. Data were analyzed using GraphPadPrism (version 4.02, GraphPad Software, San Diego, CA).

\section{Results}

\section{$\mathrm{HBP}$ is increased in plasma at onset of $\mathrm{ALI}$}

HBP concentration in plasma in healthy volunteers and patients with ALI were measured at enrollment. The median value of plasma HBP levels was $12.8 \mathrm{ng} / \mathrm{ml}$ in healthy subjects $(\mathrm{n}=5)$ and $16.7 \mathrm{ng} / \mathrm{ml}$ in patients with ALI $(n=47)$. This corresponds to a $30 \%$ increase of HBP in patients with ALI $(\mathrm{P}=0.011)$ (Figure 1$)$.

\section{HBP levels and Intensive Care Unit mortality}

We tested whether levels of HBP correlated with ICU mortality. To this end, we compared concentrations of plasma HBP in ICU survivors and ICU non-survivors at enrolment and after 3 and 7 days in ICU. At enrolment, and day 3 , there was no significant difference in plasma HBP levels between ICU survivors $(n=21)$ and non-survivors $(n=9)$ (Figure 2A \& B). However, at day 7 after enrolment, patients with ALI who did not survive had a significantly higher level of plasma HBP in comparison to ICU survivors $(p=0.013)$ (Figure $2 \mathrm{C}$ ).

The fold change in plasma HBP levels at day 7 versus day 0 was significantly lower $(p=0.022)$ in ICU survivors $(n=21)$ than in ICU non-survivors $(n=9)$ (Figure 2D). Collectively, these results indicate that ICU survival is associated with decreased plasma HBP levels at day 7 . 


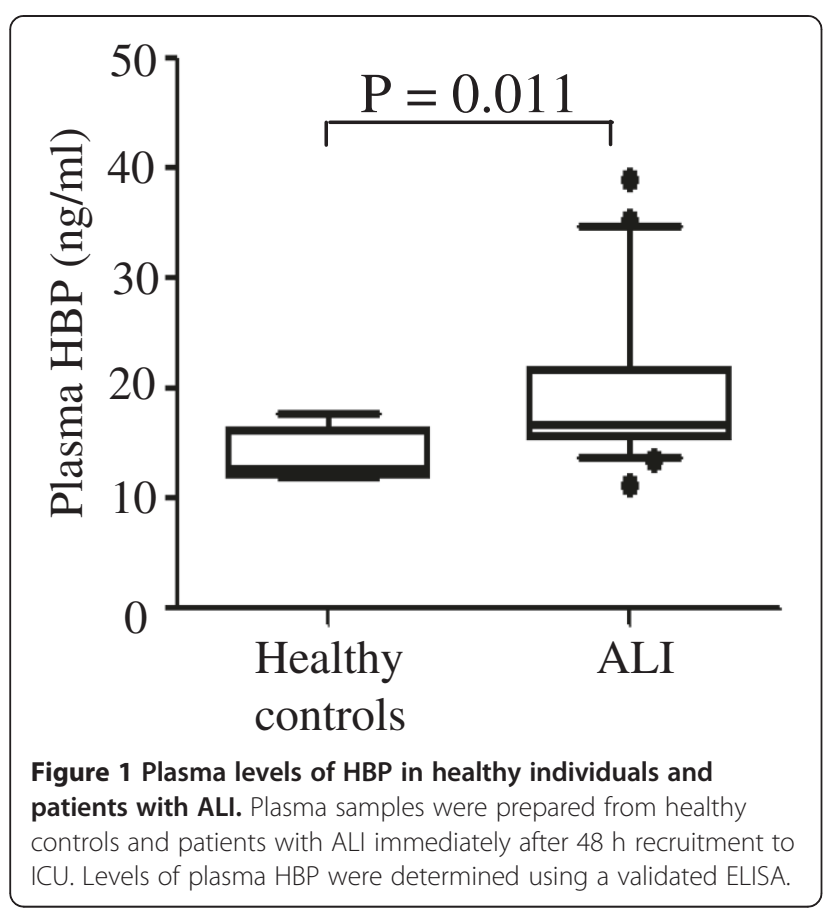

Simvastatin reduces plasma HBP levels in patients with ALI

We tested whether simvastatin could modify plasma HBP concentrations in patients with ALI. The level of plasma HBP decreased significantly at day 7 in both patients treated with placebo $(\mathrm{n}=16, \mathrm{p}=0.009)$ or simvastatin $(\mathrm{n}=12, \mathrm{p}=0.004)$ (Figure $3 \mathrm{~A})$. We found that the fold change of plasma HBP in patients with ALI treated with simvastatin was significantly lower than in placebo-treated patients $(\mathrm{P}=0.04)$ (Figure $3 \mathrm{~B})$. This result indicates that a more pronounced reduction in plasma HBP levels is observed in patients with ALI treated with simvastatin.

We corrected the concentrations of plasma HBP for the number of neutrophils in blood. When the concentration of HBP was normalized for neutrophil counts, we found no significant decrease in HBP levels at days 3 and 7 after enrolment in placebo-treated patients (Figure $3 \mathrm{C}$ ). In contrast, and similarly to above (Figures $3 \mathrm{~A} \& \mathrm{~B})$, there was a significant decrease $(\mathrm{p}=0.004)$ in HBP levels in patients treated with simvastatin at day 7 (Figure 3C). The fold change of HBP at day 7 versus day 0 in patients treated with simvastatin was significantly $(\mathrm{p}=$ 0.014 ) lower than in placebo-treated patients (Figure 3D).

\section{Discussion}

Statins may have a beneficial role in ALI. We have recently shown that oral administration of $80 \mathrm{mg}$ simvastatin to patients with ALI reduced pulmonary and systemic inflammation [14]. Simvastatin improved systemic organ failure with a trend to improvement in respiratory dysfunction [14]. Multi-organ dysfunction is caused, at least in part, by increased endothelial permeability and simvastatin has been shown to improve lung

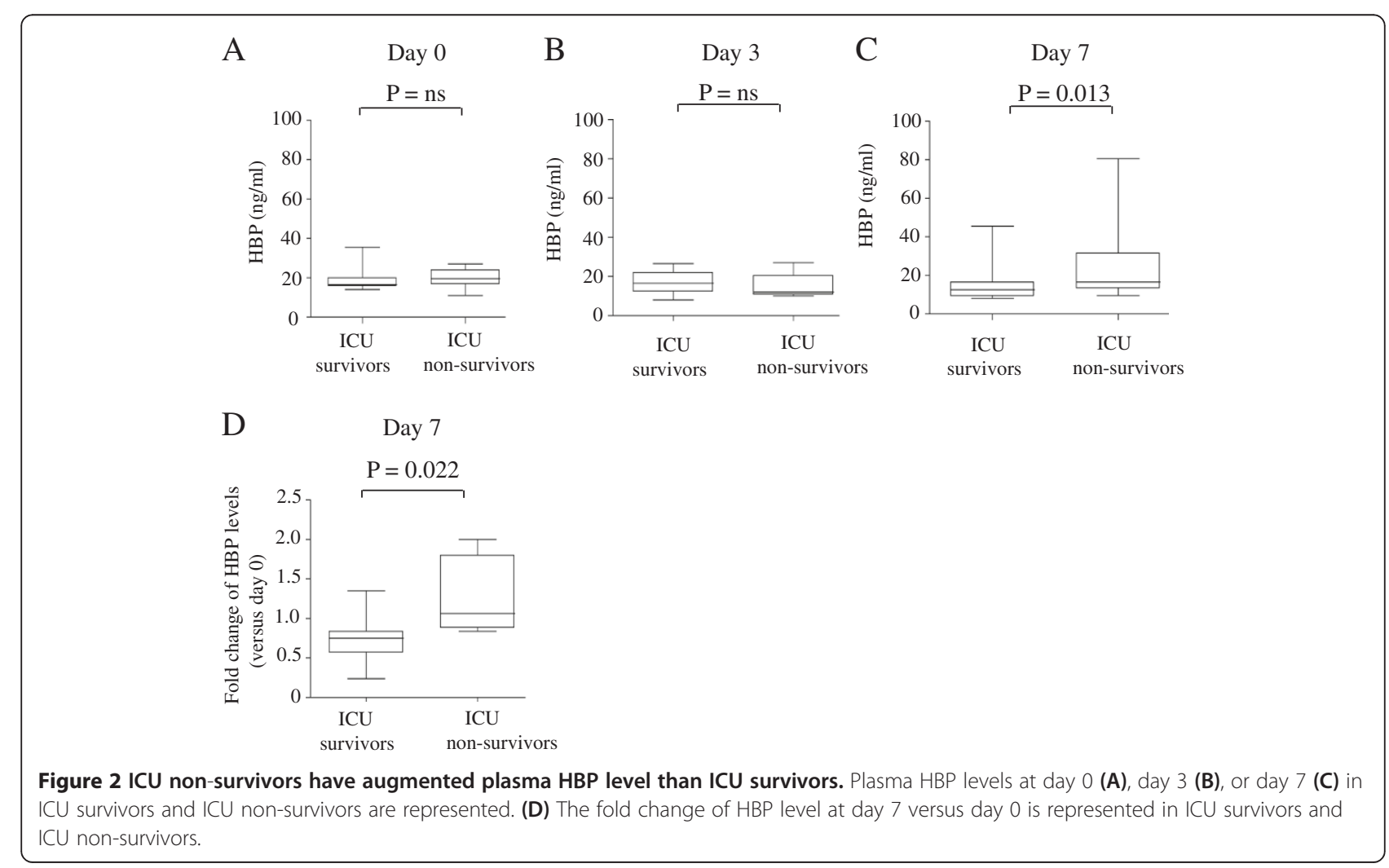




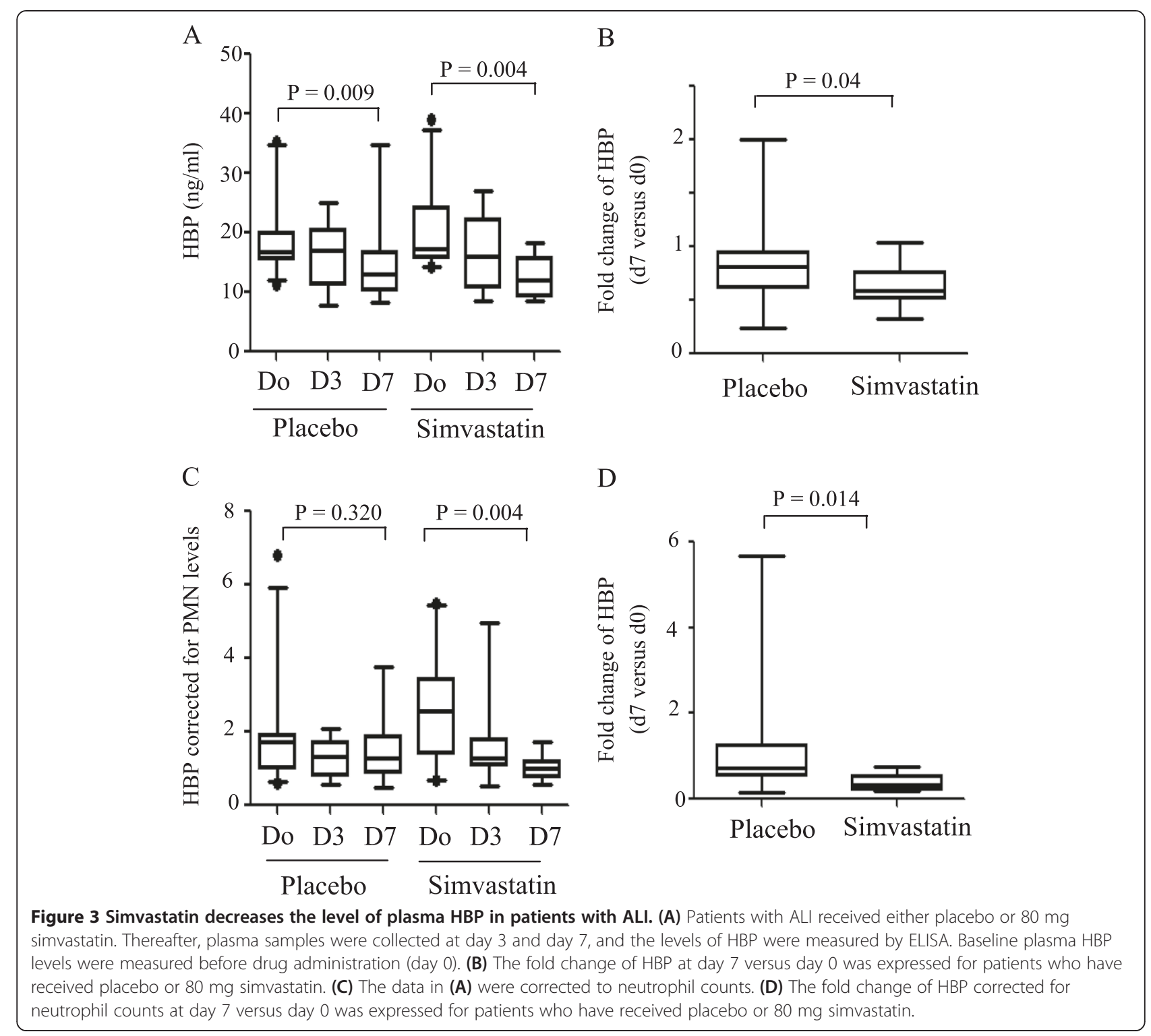

permeability in endotoxin-induced ALI in mice [15]. As HBP derived from neutrophils disrupts endothelial integrity causing vascular leakage and organ failure [4,9], we aimed to investigate the levels of plasma HBP in patients with ALI and if simvastatin modified those levels. As neutrophils play a central part in the pathogenesis of ALI [1], we hypothesised that HBP concentrations in plasma would be increased in patients with ALI. We found that plasma HBP levels in patients with ALI were significantly increased compared to healthy individuals. Other investigators found elevated levels of plasma HBP in patients with severe sepsis and septic shock $[16,17]$ and HBP concentrations higher or equal to $15 \mathrm{ng} / \mathrm{ml}$ were found in $87 \%$ of critically ill patients with sepsis. Furthermore, when plasma HBP levels reached this threshold, there was a 4-fold increase of the mortality rate among patients with sepsis [16].

We next investigated whether plasma HBP level was associated with mortality. We found that plasma HBP levels at enrolment were not significantly different between patients with ALI surviving ICU admission or not. In contrast, other investigators found elevated HBP levels at admission in other diseases including sepsis, and this was associated with an increased mortality at 28 days [16]. We also observed that HBP levels decreased significantly at day 7 versus day 0 in ICU survivors but not in ICU non-survivors. Based on these findings, we propose that normalization of plasma HBP level over the time, rather than the concentration of HBP at enrolment, is associated with recovery. It is plausible that 
strategies to decrease plasma HBP levels may reduce endothelial leak and may be a potential therapeutic strategy to further investigate.

Simvastatin reduces pulmonary and systemic inflammation in patients with ALI [14] and in a human model of ALI induced by inhaled LPS [13]. Therefore, we investigated whether simvastatin reduced plasma HBP levels in patients with ALI. We found that patients treated with simvastatin had a greater fold reduction in plasma HBP levels at day 7 versus day 0 than those who received placebo. This relation persisted when corrected for neutrophil count suggesting this is not simply a reflect of reduced peripheral neutrophil counts but reduced neutrophil activation.

The relatively small sample size used in this study limits the interpretation of the data. Although we found that decreased plasma HBP levels was associated with survival and simvastatin decreased plasma levels of HBP in patients with ALI, simvastatin did not improve ALI survival [14]. It would be necessary to conduct a larger study in patients with ALI to confirm the association between the administration of simvastatin, reduced plasma levels of HBP, and survival which is ongoing [18].

Pro-inflammatory cytokines and chemokines, which characterise ALI, induce expression of $\beta 2$ integrins (LFA-1 and Mac-1) on the membrane surface of neutrophils [19] and switch these adhesion receptors from a low to a high affinity ligand binding conformation [20]. This change in conformation allows the $\beta 2$ integrins to bind endothelial ligands, which is a prerequisite for extravasation and activation of neutrophil inflammatory functions including degranulation (and release of HBP). Interestingly, statins have been shown to bind to an allosteric site within the $\beta 2$ integrin LFA-1 which prevents LFA-1 interacting with endothelial ligands. This has been shown to impair migration of neutrophils in a murine peritonitis model [21]. Although statins do not bind Mac-1 [21], the dominant $\beta 2$ integrin expressed in neutrophils, simvastatin has been shown to reduce expression of Mac-1 on the membrane surface of circulating neutrophils by reducing the level of pulmonary CXC chemokines [22]. Accordingly, potential mechanism for our finding is that simvastatin blocks $\beta 2$ integrin-dependent release of HBP and other granule proteins.

\section{Conclusions}

This study has shown that the concentration of HBP in plasma was increased in patients with ALI compared to healthy individuals. Furthermore, we found that at day 7 after onset of ALI, high plasma HBP levels was associated with mortality. Simvastatin was associated with greater reduction in plasma HBP. A greater reduction in plasma HBP may be one of the mechanism by which simvastatin exerts a potential protective effect.

\section{Abbreviations}

ALI: Acute lung injury; ARDS: Acute respiratory distress syndrome; BAL: Bronchoalveolar lavage; HBP: Heparin-binding protein; LPS: Lipopolysaccharide.

\section{Competing interests}

The authors do not have a financial relationship with a commercial entity that has an interest in the subject of this manuscript.

\section{Authors' contributions}

DFA designed the study, analyzed the data, and contributed to the writing of the manuscript. COK contributed to the analysis of the data and the writing of the manuscript. TRC and MS collected and analyzed the data, and contributed to the writing of the manuscript. $\mathrm{HH}$ contributed to the writing of the manuscript and provided technical expertise. KD initiated the study, analyzed the data, and wrote the manuscript. All authors read and approved the final manuscript.

\section{Acknowledgements}

We thank Monica Heidenholm for technical assistance. This work was funded by the Faculty of Medicine, Dentistry, and Biomedical Sciences, Queen's University of Belfast (KD); HSC R\&D Division, Public Health Agency Northern Ireland and REVIVE, (DFA, TRC, and MS), the Faculty of Medicine, University of Lund $(H H)$, and a DH/Northern HSC R\&D Division Clinical Scientist Fellowship (COK).

\section{Author details}

${ }^{1}$ Regional Intensive Care Unit, Royal Victoria Hospital, Belfast, Northern Ireland, UK. ${ }^{2}$ Centre for Infection and Immunity, Queen's University of Belfast, Belfast, United Kingdom. ${ }^{3}$ Department of Clinical Sciences, Division of Infection Medicine, University of Lund, Lund, Sweden.

Received: 1 February 2013 Accepted: 5 July 2013

Published: 19 July 2013

\section{References}

1. Tsushima K, King LS, Aggarwal NR, De Gorordo A, D'Alessio FR, Kubo K: Acute lung injury review. Inter Med 2009, 48(9):621-630.

2. Sinclair DG, Braude S, Haslam PL, Evans TW: Pulmonary permeability in patients with severe lung injury. Clinical correlates and natural history. Chest 1994, 106(2):535-539.

3. Steinberg KP, Milberg JA, Martin TR, Maunder RJ, Cockrill BA, Hudson CD: Evolution of bronchoalveolar cell populations in the adult respiratory distress syndrome. Am J Respir Crit Care Med 1994, 150(1):113-122.

4. Gautam N, Olofsson AM, Herwald H, Iversen LF, Lundgren-Akerlund E, Hedqvist $P$, Arfors K-E, Flodgaard H, Lindbom L: Heparin-binding protein (HBP/CAP37): A missing link in neutrophil-evoked alteration of vascular permeability. Nature Med 2001, 7(10):1123-1127.

5. Tapper $H$, Karlsson A, Morgelin M, Flodgaard H, Herwald H: Secretion of heparin-binding protein from human neutrophils is determined by its localization in azurophilic granules and secretory vesicles. Blood 2002, 99(5):1785-1793.

6. Shafer WM, Martin LE, Spitznagel JK: Cationic antimicrobial proteins isolated from human neutrophil granulocytes in the presence of diisopropyl fluorophosphates. Infect Immun 1984, 45(1):29-35.

7. Soehnlein O, Zernecke A, Eriksson EE, Gigliotti Rothfuchs A, Pham CT, Herwald H, Bidzhekov K, Rottenberg ME, Weber C, Lindbom L: Neutrophil products pave the way for inflammatory monocytes. Blood 2008, 112(4):1461-1471.

8. Soehnlein $O$, Kai-Larsen $Y$, Frithiof $R$, Sorensen $O E$, Kenne $E$, Scharffetter-Kochanek K, Eriksson EE, Herwald H, Agerberth B, Lindbom L: Neutrophil primary granule proteins HBP and HNP1-3 boost bacterial phagocytosis by human and murine macrophages. J Clin Invest 2008, 118(10):3491-3502.

9. Herwald H, Cramer H, Morgelin M, Russell W, Sollenberg U, Norrby-Teglung A, Flodgaard $H$, Lindbom L, Björck L: M protein, a classical bacterial virulence determinant, forms complexes with fibrinogen that induce vascular leakage. Cell 2004, 116(February 6):367-379.

10. Fessler MB, Young SK, Jeyaseelan S, Lieber JG, Arndt PG, Nick JA, Worthen GS: A role for hydroxyl-methylglutaryl coenzyme a reductase in 
pulmonary inflammation and host defense. Am J Respir Crit Care Med 2005, 171(6):606-615.

11. Jacobson JR, Barnard JW, Grigoryev DN, Grigoryev DN, Ma SF, Tuder RM, Garcia JG: Simvastatin attenuates vascular leak and inflammation in murine inflammatory lung injury. Am J Physiol Lung Cell Mol Physiol 2005, 288(6):L1026-L1032.

12. Steiner S, Speidl WS, Pleiner J, Seidinger D, Zorn G, Kaun C, Wojta J, Huber K, Minar E, Wolzt M, Kopp CW: Simvastatin blunts endotoxin-induced tissue factor in vivo. Circulation 2005, 111(14):1842-1846.

13. Shyamsundar M, Scott TW, O'Kane CM, Craig TR, Brown V, Thickett DR, Matthay MA, Taggart CC, Backman JT, Elborn JS, McAuley DF: Simvastatin decreases lipopolysaccharide-induced pulmonary inflammation in healthy volunteers. Am J Respir Crit Care Med 2009, 179:1107-1114.

14. Craig TR, Duffy MJ, Shyamsundar M, McDowell C, McLaughlin B, Elborn JS, McAuley DF: A randomized clinical trial of hydroxymethylglutaryl-coenzyme A reductase inhibition for acute lung injury (The HARP study). Am J Respir Crit Care Med 2011, 183:620-626.

15. Grommes J, Vijayan S, Drechsler M, Hartwig H, Mörgelin M, Dembinski R, Jacobs M, Koeppel TA, Binnebösel M, Weber C, Soehnlein O: Simvastatin reduces endotoxin-induced acute lung injury by decreasing neutrophil recruitment and radical formation. PLoS One 2012, 7(6):e38917.

16. Linder A, Akesson P, Inghammar M, Treutiger C-J, Linnér A, Sundén-Cullberg $\mathrm{J}$ : Elevated plasma levels of heparin-binding protein in intensive care unit patients with severe sepsis and septic shock. Crit Care 2012, 16:R90.

17. Chew MS, Linder A, Santen S, Ersson A, Herwald H, Thorlacius H: Increased plasma levels of heparin-binding protein in patients with shock: a prospective, cohort study. Inflamm Res 2012, 61(4):375-379.

18. McAuley DF, Laffey JG, O'Kane CM, Coss M, Perkins GD, Murphy L, McNally C, Crealey G, Stevenson M, HARP investigators; Irish Critical Care Trials Group: Hydroxymethylglutaryl-CoA reductase inhibition with simvastatin in acute lung injury to reduce pulmonary dysfunction (HARP-2) trial: study protocol for a randomized controlled trial. Trials 2012, 13:170. doi:10.1186/1745-6215-13-170.

19. Sengelov H, Kjeldsen L, Diamond MS, Springer TA, Borregaard N: Subcellular localization and dynamics of Mac-1 (alpha m beta 2) in human neutrophils. J Clin Invest 1993, 92:1467-1476.

20. Diamond MS, Springer TA: A subpopulation of Mac-1 (CD11b/CD18) molecules mediates neutrophil adhesion to ICAM-1 and fibrinogen. J Cell Biol 1993, 120:545-556.

21. Weitz-Schmidt G, Welzenbach K, Brinkmann V, Kamata T, Kallen J, Bruns C, Cottens S, Takada Y, Hommel U: Statins selectively inhibit leukocyte function antigen-1 by binding to a novel regulatory integrin site. Nature Med 2001, 7:687-692.

22. Zhang S, Rahman M, Zhang S, Qi Z, Herwald H, Thorlacius H: Simvastatin regulates $\mathrm{CXC}$ chemokine formation in streptococcal $\mathrm{M} 1$ protein-induced neutrophil infiltration in the lung. Am J Physiol Lung Cell Mol Physiol 2011, 300:L930-939.

doi:10.1186/1471-2466-13-47

Cite this article as: McAuley et al:: Simvastatin decreases the level of heparin-binding protein in patients with acute lung injury. BMC Pulmonary Medicine 2013 13:47.

\section{Submit your next manuscript to BioMed Central and take full advantage of:}

- Convenient online submission

- Thorough peer review

- No space constraints or color figure charges

- Immediate publication on acceptance

- Inclusion in PubMed, CAS, Scopus and Google Scholar

- Research which is freely available for redistribution 